percentage of 12 to 15 is nearer the truth." Contrast this with the 57 per cent. of cures which I have obtained with the more thorough operation.

I have previously mentioned several cases in which I do not consider that a radical operation is advisable, and the question in these is whether anything can be done in the way of prolonging the life of the patient, or of making the period of life that remains to her more comfortable. In some of these cases the patient's life can no doubt be prolonged by excision of the breast alone, or of the axillary glands as well, perhaps for three or four months or even as long as a year, and she may then die of internal disease without suffering so much as she would have done from the local tumour. She should, however, be told that the object of the operation is merely to prolong life, and many women, knowing that, would prefer not to undergo it. In some cases where there is a foul ulcer the patient may be relieved of much pain and trouble by removal of the breast. On the whole, however, I do not see any particular advantage in operating in cases of breast cancer unless with the view of curing the disease, and in cases where it is practically certain that the disease cannot be cured the performance of an operation would depend entirely upon the patient's desire for a short prolongation of life or on the possibility of relieving her of some serious trouble. As a matter of fact, we do not have here, as we have in intestinal cases, any substitute plan to offer which will either relieve the patient materially or give any marked prolongation of life. It is practically either a thorough operation or nothing, and therefore the breast is not a situation in which the so-called careful selection of cases and exclusion of all but simple cases from operation is at all permissible.

\section{THIRTEEN CASES OF STRANGULATED OVARIAN CYSTS.}

BY HARRISON CRIPPS, F.R.C.S. ENG.,

ASSISTANT SURGEON TO ST. BARTHOYOMEW'S HOSPYTAL AND OPERATOR FOR THE ABDOMINAI SECTYONS IN THE WOMEN'S WARD.

THE frequency with which cysts may become twisted on their pedicle can be gathered from the fact that during three years $I$ have met with thirteen cases in the women's ward at St. Bartholomew's Hospital, and this out of a total of a little over 100 operations for ovariotomy during that period. In the majority of instances the onset of the symptoms suggesting strangulation was sudden, in the others it was rather of a subacute nature. The symptoms of this accident are so suggestive and typical that there ought to be little difficulty in making an accurate diagnosis. The condition, if left unrelieved, though not necessarily fatal, is one of extreme danger, while with timely surgical aid life is almost invariably saved and the patient completely cured of her trouble. The effect of the twist varies somewhat according to its tightness and the nature of the cystic contents. If the twist be only partial the veins, owing to the comparative thinness of their walls, become obstructed, while the arteries still continue to pump blood into the tumour. The effect of this is so to increase the blood-pressure in the tumour and its capsule that the capillaries or thin-walled veins give way, and hæmorrhage takes place both within the tumour and its capsule. In this way $\bar{a}$ very considerable hæmorrhage may occur, the cysts becoming filled with blood, while a large hamatoma may be formed in the capsular connective tissue. It would seem, however, that although the stoppage of the circulation at first takes place from compression of the veins, that stasis soon occurs and the blood clots in the supplying arteries as well as in the effluent veins. In some cases from the first the twist is so complete as at once to cut off all blood supply to the tumour. When the twist is sufficiently tight to impede or cut off the blood supply secondary changes occur with more or less rapidity. It would seem from the study of the cases recorded that it is quite possible to have a twist of the pedicle giving rise to sudden and acute symptoms, which nevertheless may relieve itself by again becoming untwisted and allowing the engorged veins to empty themselves. This is what must have occurred in Case 12. The two previous attacks to the one for which the patient was admitted must have been almost certainly due to a twist which was thus relieved before the blood had permanently clotted in the veins. Very soon after a twist has occurred, and probably coincident with total cessation of the circulation within it, nature seems to regard the cyst as a foreign body and makes every effort to surround and encase the tumour with inflammatory exudation. This exudation is of course poured out, not by the dead tumour, but by the living tissues in contact with it. Thus the parietal peritoneum, the intestines, the uterus, bladder, omentum, in fact, every tissue in contact with the tumour, becomes adherent to it by the exudation of plastic lymph. If the tumour happens to be small and its contents simple cystic fluid the cyst wall obtains sufficient nourishment from these adherent structures to maintain its vitality. On the other hand, if the cyst be large, and if its contents are already purulent, or if there be much extravasated blood within it, the efforts of the neighbouring tissues to adhere to and nourish it are ineffectual, and the whole cyst wall falls quickly into a state of gangrene, or it may more slowly soften in parts and give way, allowing the contents to be extravasated. Whether the wall simply becomes gangrenous or whether the contents are extravasated as well acute peritonitis with death is the result.

With regard to diagnosis, the fact that a cyst can become twisted points to its having a long and comparatively narrow pedicle. It must be practically free from adhesions. It cannot be beneath the broad ligament, and must be well out of the pelvis, and it is just this form of tumour which, in an early stage and whilst small, gives so little inconvenience that a patient may be quite unaware of its existence until a sudden onset of a characteristic symptom of twisting (see Case 13). In the majority of cases, however, the patient will be aware that she previously has had a tumour, though it may have caused little trouble. If the twist occur suddenly the symptoms are very characteristic. The woman being previously in comfort is suddenly seized with severe abdominal pain, sometimes sufficient to make her feel faint and generally followed by some vomiting. There is a diffuse tenderness over the whole abdomen. The patient, if she knows that she has previously had a tumour, will generally say that coincident with the attack the tumour seemed to swell up and become harder. On abdominal examination the tumour may be felt of a globular outline, but generally of not a very large size. It is tender on pressure, and has a somewhat firmer feel than the majority of ovarian cysts. It can be moved from side to side as if rocking on a long pedicle, but nevertheless it does not seem to rock independently of the parietal peritoneum to which it is often slightly adherent. Occasionally some creaking can be heard and felt due to the rubbing of inflamed peritoneal surfaces (see Cases 7 and 8 ). Per vaginam the uterus is probably movable and the tumour, if felt at all, is lying higher up, only resting on the brim of the pelvis. The patient's general condition is one of distress. She suffers from pain and uneasiness, constipation, and not infrequently retention of urine, the pulse is rapid, and the temperature more often raised than not, though here, as in other abdominal cases, the thermometer is an unreliable guide. It has this advantage, however, that with severe general symptoms, if the temperature is raised the prcgnosis is far more favourable than when the same symptoms are present with a normal or subnormal temperature. The symptoms somewhat resembling those of a twisted cyst may be due to a simple inflamed cyst, a ruptured cyst, an extra-uterine foetation, or a hæmatocele. It is not possible to diagnose whether a cyst is inflamed from a twist or other causes, nor is it important, for in either case an operation should be undertaken. If the symptoms be due to the rupture of a cyst there will be the same sudden symptoms as if a twist had occurred, but there is often this important difference, that the outline of a twisted cyst is well defined, feels firm, and the tumour is somewhat larger than previously, while in a ruptured cyst the outline of a tumour becomes indistinct and feels flaccid, is smaller, or even disappears, whilst there is dulness in the flanks from gravitation of the extravasated contents to these regions. When the symptoms are due to a ruptured extra-uterine fotation there will of ten be a history of missed, or at least irregular, periods. There may be suggestive signs about the breasts. The pain is more pronounced, while it is almost invariably accompanied by fainting and other symptoms of collapse. The anæmic condition of the tissues, soft, rapid pulse, and sighing respiration suggest bleeding, while an abdominal examination shows there is no definite tumour. 
These symptoms collectively can scarcely be mistaken for a twisted cyst On the other hand, a limited hrematocele into the broad ligament or into Douglas's pouch may closely resemble a twisted cyst. There was such a case recently in the Martha Ward at St. Bartholomew's Hospital. In this instance a tumour was supposed to have existed for some time, but acute symptoms had only been present for three weeks. A hard, firm tumour could be felt rising half-way to the umbilicus, well defined and very tender, but the uterus was fixed and the tumour extended down into Douglas's pouch. On operating the tumour was found to consist of blood clots the size of a child's head in Douglas's pouch, spreading upwards in a globular form, being roofed in by inherent omentum and intestines. In such a case the tumour in Douglas's pouch and the fixing of the uterus should have suggested the nature of the case. If a twisted cyst is not diagnosed early the symptoms may rapidly pass on into those of acute peritonitis, and owing to the distension of the intestines disguising the cyst the diagnosis is obscure.

As to treatment, this, fortunately, is very simple, for directly the nature of the case is suspected the abdomen should be opened and the cyst removed. The fact that the tumour has become twisted shows that it has a long pedicle, that it is well out of the pelvis, and can have but slight adhesions, conditions which make the removal of any ovarian cyst particularly favourable. What the surgeon is anxious about is that the operation should be undertaken before the occurrence of acute peritonitis. Should this have occurred it is difficult to save the patient. In such a case, if not actually moribund, the abdomen, after removal of the tumour, should be thoroughly flushed and subsequently drained. Death occurred in only one of the cases recorded. In this instance the delay had been too long, the cyst had burst, and at the time of the operation there was acute general peritonitis. The majority of the cases were admitted under the care of my colleagues, Dr. Champneys and Dr. Griffith, and were transferred to me for operation.

CASE 1.-A woman aged forty-four years who was admitted to hospital in August, 1892. For seven years she had noticed a swelling in the abdomen, which during the last few months had got considerably larger, but it did not give her much pain or inconvenience. On Aug. 18th, whilst washing, she was suddenly seized with acute pain in the abdomen which made her feel faint. From that time till she was admitted, four days later, she had been ill with considerable pain and for the last two days there had been some vomiting. On admission she was found to be much collapsed, with a scarcely perceptible pulse at the wrist. The abdomen was opened and a large multilocular cyst found with slight recent adhesions all over the surface to the parietes. Here and there some yellow lymph on the surface was found, and also in one or two places on the intestines. The tumour was removed and the abdominal cavity washed, but the patient practically never came out from the shock from which she was suffering prior to the operation and died a few hours later. The cyst was twisted one and a half times on its axis.

CAsE 2.-A woman aged twenty-four years who was admitted to hospital in February, 1893. She had noticed a lump in her abdomen for three or four months, but it had given her very little pain, and she had seen her last period a week ago, since which time she had had great pain and she thought the tumour had got somewhat larger. On admission there was a tumour extending as high as the costal cartilages on either side, feeling very tight, with considerable tenderness on pressure. The patient's temperature was somewhat raised and she had a pulse of 120 but of fair volume. On opening the abdomen the tumour proved to be a large multilocular cyst with one complete twist in its pedicle. It was adherent by recent adhesions to the parietes and omentum. After removal it was found that there had been considerable hæmorrhage into the cyst and some small hæmorrhagic nodules in the cyst wall. The abdominal cavity was flushed and the drainage tube left in for a few days. The patient recovered.

CASE 3. - A woman aged forty-one years, who was admitted to hospital in March, 1893. She had been in the hospital four years previously suffering from an abdominal tumour which then caused some difficulty in micturition. Since that time, although the tumour had increased somewhat in size, it had given her no pain, and she was able to do her work. A week previously abdominal pain suddenly came on and was followed by constipation. She had no vomiting and the pain was of a spasmodic character; the abdomen was extremely tender. On examination a fuctuating swelling could be felt-dull on superficial percussion, resonant on deep percussion-occupying the lower half of the abdomen. She could scarcely bear pressure, all the parts being exquisitely tender. The temperature was not raised, but the pulse was quick. On opening the abdomen a broad ligament cyst on the right side was found with one and a half complete twists at its pedicle. It contained blood-stained fluid, and there were some clots situated between the cyst wall and the peritoneum. The patient recovered.

CASE 4.-A woman aged twenty-five years, who was admitted to hospital in February, 1894. She had noticed a tumour for nearly a year and a half; at one time it got verv much smaller, but it increased again six months ago. She thought it had increased considerably during the last two months, and for five weeks she had had a considerable amount of pain. On examination a large egg-shaped, very tender tumour could be felt, extending as low as Poupart's ligament on each side and up to the costal arches. On opening the abdomen the tumour was found to contain a dirty red-coloured fluid, about ten pints of which was drawn off, consisting largely of blood. The tumour was found to have a complete turn from left to right at its pedicle. There were also some old hæmorrhages in the sac of the cyst. The patient was seriously ill for some days after the operation with a good deal of vomiting and hiccough, but ultimately made a good recovery.

CASE 5.-A woman aged forty-one years, who was admitted to hospital in February, 1894. She had noticed the tumour for about a year, out it did not give her much trouble till four days previously, when whilst at work she was attacked by severe pain described as "labour pains." These pains continued more or less ever since, and she had a good deal of vomiting, and said the tumour felt much harder and a little larger than it was before the attack. On examination in the right half of the abdomen, extending about two inches abore the umbilicus, was a firm, elastic tumour, very tender on pressure and giving the idea of tightly compressed fluid. Per vaginam the tumour could not be felt. On opening the abdomen a considerable quantity of blood. stained fluid escaped from the peritoneal cavity. A large cyst, dark-coloured on the surface, then came into view. It was found that it had one complete twist at the pedicle. There was no adhesion anteriorly, but on raising the tumour the sigmoid flexure of the colon was found to be somewhat firmly fixed to it on the left side by old adhesions, which took some trouble to separate. No flushing or drainage was employed. The patient recovered.

CASE 6. - A woman aged forty-four years, who was admitted to hospital in March, 1894. She stated that she had noticed her abdomen getting larger for the last year and a half, but it gave her no trouble till a month previously, when for the first time she had pain. The pain had continued more or less severe since, and during this time she had many attacks of retention of urine. The abdomen was much distended by an elastic and very tender tumour rising to the costal arches. Per vaginam the tumour could just be felt lying on the brim of the pelvis. On opening the abdomen a multilocular cyst of the left ovary was found. It was slightly dark in colour on the surface and had little more than a complete twist at the pedicle. The main cyst contained a dark-coloured fluid of the consistency of glycerine. Many of the secondary cysts, however, seemed to be distended by a simple blood-clot. There were a few recent adhesions to the parietal peritoneum. Owing to a considerable amount of the fluid escaping into the peritoneal cavity it was well flushed with water, but no drainage was used. The patient recovered.

CASE 7.-A woman aged twenty-two years, who was admitted to hospital in March, 1894. She had noticed her abdomen getting large for a twelvemonth, and had had occasionally slight pain, but nothing to speak of. Two days previously she had a sudden attack of violent pain lasting four or five hours, and she romited two or three times. The pain then got better, but continued in a modified form till her admission. On admission she had a good pulse and a temperature of $101^{\circ} \mathrm{F}$. The lower abdomen was occupied by a spherical tumour arising within three inches of the ensiform cartilage. It felt densely hard, not quite globular in outline, and with one big projection on its left side. A well-marked crepitus could be felt and heard over its surface when she took it deep breath. Per vaginam, the tumour could just be felt lying on the brim of the pelvis. On opening the abdomen the cyst was found to be multilocular, containing a great 
deal of apparently solid growth which, however, afterwards turned out to be chiefly blood-clot. It could not be reduced by tapping, so the incision was enlarged to eight inches, the whole tumour being turned out. It was found to have one complete twist at the pedicle. No flushing or drainage were employed. The patient recovered. A year later I had the following interesting letter kindly written to me by $\mathrm{Dr}$. W. E. Porter. He said: "The girl aged twenty-two years, upon whom you operated in March, 1894, was delivered of a fine, healthy child on March 15th, 1895, that is, just a year after the operation, and she made a very good recovery. I was anxious about her because eighteen months ago I attended a lady who had been operated upon ten years previously for ovariotomy, and who some three months before her full term of pregnancy was finished died suddenly of internal hæmorrhage, the result, probably, of the tearing of some of the old adhesions."

CASE 8.-A woman aged fifty-eight years, who was admitted to hospital at the end of March, 1894. For the last six or eight months she had noticed a hard lump in the right iliac fossa, but it had never given her much trouble. Three weeks ago, however, she had very severe pain in the neighbourhood of the tumour and noticed that the tumour seemed to have increased slightly in size and had become very hard. On admission, the temperature was not raised, and she had a good pulse. On examining the abdomen a tumour extending to the costal arches and feeling very firm was found. It was very irregular on the surface and well-marked crepitus could be heard and felt over portions of the tumour. Per vaginam a part of the tumour could be felt lying behind the uterus. The abdomen was opened by a four-inch incision, and a moderate amount of blood-stained fluid escaped from the peritoneal cavity. On the surface of the tumour was a small quantity of lymph in places. The tumour was composed of many small cysts. A trocar was put in, but practically no fuid came out. The incision was then prolonged and the tumour removed entire. It was found on section that most of the secondary cysts contained blood. The abdominal cavity was flushed but not drained. The patient recovered.

CASE 9. - A woman aged thirty-eight years, who was admitted to hospital in July, 1894. She had noticed herself getting larger for nearly two years, but much more rapidly during the last three months. She was a healthy-looking woman, and had not complained of much pain. On examination the abdomen was found to be distended by an irregular tumour, extending to midway between the ensiform cartilage and the umbilicus. The consistency varied in different parts, some being elastic and apparently fluctuating, while others were quite firm and hard. Per vaginam the sound passed two inches and a half and the uterus was fairly movable; the tumour could be felt lying on the brim of the pelvis. On opening the abdomen the tumour proved to be a multilocular ovarian cyst in some portion of which were hard nodules. It was slightly cedematous, but there was no hxmorrhage into it or into its capsule; it was twisted half a turn to the left. The tumour consisted of the right ovary. There were no adhesions. No irrigation or drainage was employed. The patient recovered.

CASE 10.- $A$ woman aged twenty-five years, who was asmitted to hospital in August, 1894. About a year ago she first noticed sharp pain in the groin. On admission the patient did not feel ill, but there was much tenderness over the abdomen. Examination showed a tumour extending half way between the pubes and the umbilicus; it was slightly movable and gave an elastic sensation. Per vaginam the tumour could be felt high up in the utero-vesical pouch the sound passed three and a half inches. On opening the abdomen the tumour proved to be a dermoid cyst of the left ovary. There were no adhesions. It was found to have had one and a half complete turns of the pedicle. On examining it after removal it contained large quantities of sebaceous material, hair, teeth, and bones, but no blood. No irrigation or drainage was employed. The patient recovered.

CASE 11. - A woman aged thirty-one years, who was admitted to hospital in August, 1894. She noticed after her last confinement, thirteen months previously, that the abdomen did not seem to regain its natural size, and during the last two or three months had been getting considerably larger, but she had had no pain and no trouble with the urine, though she had been getting thinner. On admission the abdomen was found uniformly distended by a tumour extending to within two fingers' breadth of the costal arches on either side. Some portions of the tumour felt hard and others comparatively soft, but owing to a good deal of fat in the abdominal walls it was nct easy to determine the exact consistency. Per vaginam, the os was found dilated, and would admit the finger. The uterus was freely movable. No part of the tumour could be found in the pelvis. The sound passed two inches and a half. The tumour proved to be a multilocular cyst of the left ovary. Its contents were very thick and would not run through the trocar. A certain amount of the contents were extravasated into the abdominal cavity during the removal of the tumour. There was half a twist of the pedicle. The abdomen was well irrigated to wash out the material that had escaped during the extraction of the cyst. No drainage was used. The patient recovered. An interesting point about this case was the loss of one of the sponges. On counting the sponges after the operation it was found that one of them, a thin flat sponge five inches in diameter, was missing. A thorough search in every direction was made for it without result. It could not be found in the abdominal cavity. The nurse then stated that during the operation she observed at one time that the water did not run very freely away from the basin where she was washing the sponges. The opening at the bottom of the basin was only three-fourths of an inch in diameter, and it did not look as if it was possible for a sponge to pass through. However, the plumber was sent for, and, the force pump being used, the sponge was expelled into the trap at the base of the building. It was almost impossible to conceive that this large sponge could pass through so small an opening. Indeed, as we endeavoured to force it down subsequently it was quite impossible to get it through, but when smeared with some of the mucus of the tumour it was possible to pass it through the hole at the bottom of the basin with comparative ease. Since this accident a fine wire grating is put down the hole in the bottom of the basin and I never allow any water to be thrown away except through this, so that it is absolutely impossible that such an accident should occur again.

CASE 12. - A woman aged thirty-three years who was admitted to hospital in October, 1894. Three weeks after her last confinement, thirteen months previously, she was suddenly seized with severe abdominal pain, but in a couple of days she was all right again. Since that time she has had four or five similar attacks, lasting from a few hours till a couple of days. She had also noticed during the whole of this time a swelling in her abdomen. She was very clear that at the time of these attacks the swelling appeared to get larger and was very tender. She came to the hospital during one of these attacks suffering intense pain, and was at once admitted to the Martha Ward. On examining the abdomen the tumour was found to be about two inches above the umbilicus. It was slightly displaceable from side to side and rather tender upon pressure. Per vaginam high up a rounded swelling could be felt in front of the uterus. Bimanually a tumour could be felt which apparently was about the size of a six months pregnancy. On opening the abdomen a tense, dark-bluish, grey tumour presented which was brought out entire after enlarging the wound. The pedicle and tumour were free from adhesions. It was found twisted one and a half times in the same direction as a corkscrew. Some fluid from a cyst in it had ruptured and escaped into the peritoneal cavity, which was thoroughly washed out with sterilised water. The wound was entirely closed without drainage. The tumour proved to be a multilocular cyst of the right ovary which was twisted on it pedicle. It contained a large quantity of dark, chocolatecoloured blood. There were several secondary cysts all containing blood-clot or fluid blood. The patient recovered.

CASE 13. - This patient was a woman aged thirty-eight years who was admitted to hospital in April, 1895. She had eleven children, the youngest born fifteen months previously, which she was still suckling. She said she had never got to her proper size since her last confinement. Six months ago she had some pain in the left groin, and had loss of flesh and felt ill since. Fourteen days before admission she was suddenly seized with severe pain in the left side, and for the first time she then noticed a lump in this position. On admission there was a fluctuating tumour rather firm extending midway to the ensiform cartilage. It was altogether above the pelvis. Per vaginam the cervix was very soft and the vagina mauve in colour, and an impulse was easily conveyed from the tumour to the cervix and there was some doubt as to whether the patient might not be pregnant. The evening before the operation the abdomen was noticed to have lost a good deal of its disten sion, the tumour being much more ill-defined than it was at 
first. An incision was made in the middle line from the umbilicus downwards for three inches, which was subsequently enlarged a couple of inches downwards. On opening the peritoneal cavity a considerable quantity of mucopurulent fluid escaped. This was found to be the contents of a ruptured cyst. The whole tumour, which was growing from the left ovary, was brought forward and was found to be twisted one complete turn at its pedicle. The main mass was pulled out of the abdominal cavity, the rent in the cyst enlarged, and the cavity emptied, the secondary cysts also being emptied from the same opening. The pedicle was then transfixed in the usual way. The abdominal cavity was tery thoroughly washed out with sterilised water. There was no drainage. The patient recovered.

\section{A RECORD OF THE COMMON SORE- THROATS OCCURRING AMONGST 400 ADOLESCENTS DURING A PERIOD OF TWENTY-FIVE YEARS.}

BY CLEMENT DUKES, M.D., B.S. I,OND., EHYSICIAN TO RUGBY SGHOOL AND SENIOR PHYSICIAN TO RUGBY HOSPITAI.

IT seems to me to be the duty of those members of the profession who occupy positions where exceptional opportraties occur to recount their facts from time to time. With this feeling in view I place on record these few observations. It is necessary to explain a little in detail the system of work in force so as to ensure the value of the facts. During the whole of the twenty-fire years I have been physician to the Rugby School I have visited with the utmost regularity every boarding-house where the members of the school reside, starting at exactly the same time $(8.15 \mathrm{~A}$.M.) every week-day during every term, and on Sundays at $8.45 \mathrm{~A} . \mathrm{M}$. The members of the school are housed in nine boarding-houses, and the numbers, varying from year to sear, give an average of just over 400 ; at the present moment they are 500. In consequence of this reguLarity and by the use of a little moral persuasion, both with regard to each house-matron and the boys themselves, $I$ am usually able to see the beginnings of all illnesses; in fact, I doubt whether anyone, except where the same system is in force, has ever had such a unique opportunity, amongst such a number, and extending over so many years. I am continually instilling into the matrons and the boys the importance of my seeing invalids in the very earliest stage of not feeling well, hours before one can definitely diagnose the ailment which is causing the discomfort. In this way I am able not only to learn the symptoms of the onset of disease, but, a circumstance of still greater moment to the individual patient, to place him early under the most favourable conditions as regards limself, and to isolate him if need he for the protection of school-fellows. In my very early days of office here I recognised the importance of knowing the condition of the throat in the young, it being involved in so many illnesses ; consequently the habit arose intuitively of looking at the throat of every boy who expressed himself as not feeling well, without waiting for any complaint from in concerning it. And whenever I noticed the least deviation from a healthy appearance, however trivial, recognising the fact that everything has a beginning, strict isolation was enforced, and he was kept away from the healthy until quite recovered. In this way all ailments attended with sore-throat, some of them of the greatest moment to the young, have been prevented from spreading. I scarcely need to point out that it is the first cases that one requires to be always on the look out for and to isolate if the diseases are to be limited in extent, as I think I shall prove that they can be. On inspecting the throat in the young in the very earliest stage of its abnormality certain common conditions are discovered which gradually assume the various forms typical of recognised diseases. And I see these throats and isolate them often long before a diagnosis is possible. When asked, as 1 frequently am, what is the matter with the throat I am only able to plead ignorance; but I strive to impress the fact upon my inquisitors that when I am able to diagnose the case at first sight I have secured it far too late for the welfare of the individual and for the protection of other members of the school.
1. Inflammatory sore-throats.-The first stage of all the common sore-throats, which are termed "tonsillitis," is that the membrane of the fauces generally appears smooth, dry, glistening, and as if all secretion and the superficial layer of the mucous membrane had been scraped off and the surface polished. Many throats under treatment proceed no further than this stage. In the next condition this superficial layer of the mucous membrane is swollen, which causes it to assume a dusky-red velvet appearance, and this also may proceed no further. This is succeeded in a large proportion of cases by swelling of the tonsils, which gradually become covered with minute isolated white dots, and which are occasioned by an excess of the normal secretion, owing to the inflammation, from the follicles of the tonsils. This secretion in certain cases, and these individuals usually have this kind of throat whenever they take cold, becomes so excessive as not only to appear at the orifices of the follicles, but the white spots join one another until the surface of the tonsil becomes covered with a thick, soft, ashy grey, dirtylooking membrane, but which bears no more relation to diphtheria than chicken-pox does to small-pox. The same individual invariably has the same kind of inflammatory throat, and whenever he takes cold it is in the form of, or accompanied by, a sore-throat. Some of these boys have a sore-throat at least once a term, and as some remain at school several years the same individual is accounted for many times over in my list of cases. In the last phase of these cases of tonsillitis the inflammation and swelling-often after a prior subsidence, so as to seem almost like a recrudescenceincrease, extend, and involve the soft palate, and a quinsy is formed. I regard all these forms of tonsillitis as infectious in the young, and they spread in the same way, but not to the same extent, as common colds.

2. Septic sore-throats.-As our water-supply, house drainage, and town drainage are good, the septic sore-throats which arise from defects in these arrangements do not come under my notice in relation with the school. I may mention that I am very importunate over the question of flushing drains and emptying druking-water cisterns and filters before the reassembling of the school after each vacation. As further evidence ef the sanitary state of the school premises, I would lay stress upon the fact that during twentyfive years there have only been four cases of typhoid fever in the school, three of which I traced to their origin. One occurred in 1871, its origin was untraced; another appeared in 1877, from a recognised defective drain which was not remedied as soon as it should have been; another occurred in 1887, through an enthusiastic naturalist being allowed by his parent as a great treat to have a day in Cambridgeshire prior to his return to school to catch swallow-tail butterflies, and, being very tired and parched with thirst, he lay on his stomach and drank from a ditch, having only just time to catch his train ; the last, which occurred in 1889 from eating oysters, was, unfortunately, fatal.

3. The sore-throat of scarlet fever in its initial stage consists of a diffuse, dull, brick-red appearance of the mucous membrane of the fauces-in fact, it is a diffuse rash on the mucous membrane similar to that which occurs subsequently on the skin. It commences at, or at all events is most visible on, the wings of the soft palate extending to the tip of the uvula, but it involves the whole of the mucous membrane of the fauces. In a large proportion of cases it begins and ends in this, though in many it progresses to every phase of ulceration, which alone is sometimes difficult to assign to scarlet fever; but the character of the rash, together with the peeling tongue on the fourth day, leave little doubt as to the diagnosis. It is the slight sore-throat accompanied by merely a transient rash which causes the main difficulty in diagnosis.

4. The sore-throat of diphtheria.-At the onset there is usually a dark redness and swelling of the tonsils and the fauces generally, with very marked cedema of the uvula, which appearances are quite undistinguishable from acute tonsillitis. Hence the necessity of at once isolating all cases of tonsillitis, as no one can possibly be sure what form of sore throat it will prove to be. Subsequently the whole, or part, of the fauces becomes covered with a greyish secretion, sometimes assuming the character of a dense membrane like wet wash leather, which arises in patches, and rarely in the small white regular dots which coalesce into a patch incidental to tonsillitis. These patches spread and may become continuous involving the whole of the fauces.

5. The sore-throat of varicella is occasioned by spots on the mouth and pharynx similar to those on the skin, and they 